\title{
Numerical simulation of beam propagation in a layer filled with chiral nematic liquid crystals
}

\author{
Filip A. Sala* and Mirosław A. Karpierz \\ Faculty of Physics, Warsaw University of Technology, Koszykowa 75, 00-662 Warszawa
}

Received November 22, 2009; accepted December 22, 2009; published December 31, 2009

\begin{abstract}
Simulation of light beam propagation in chiral nematic liquid crystals, which are anisotropic media, requires the use of fullvector methods. One of them is FV-BPM (Full-Vector Beam Propagation Method). In the present work an application of FV-BPM to simulate light propagation, in the linear case, is shown. Calculations are carried out for various values of incident beam full width at half maximum, position of the launched beam and the pitch of the cholesteric
\end{abstract}

Chiral nematic liquid crystals are the anisotropic media. As the pitch of the cholesteric is much greater than the wavelength, the birefringence axis has a different direction along the structure so scalar methods are not suitable. Only vectorial methods can be used for proper numerical simulations of light propagation in such structures[1]. One of them is FV-BPM (Full- Vector Beam Propagation Method). This method can be, for instance, used for determining parameters for which solitons[2-5] (in NLCs called nematicons[6]) are formed or to simulate light propagation in liquid crystalline waveguides.

The algorithm bases on Maxwell equations:

$$
\nabla \times \vec{E}=-\mu \mu_{0} \frac{\partial \vec{H}}{\partial t} \quad \nabla \times \vec{H}=\varepsilon \varepsilon_{0} \frac{\partial \vec{E}}{\partial t}
$$

We assume complex electric and magnetic fields.

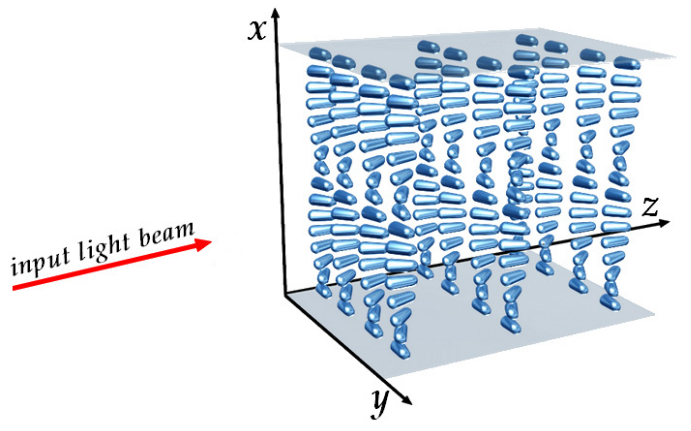

Fig. 1 Analyzed structure

\footnotetext{
*E-mail: sala@if.pw.edu.pl
}

In our work, chiral nematic liquid crystals are analyzed. Such structures have fixed anchoring conditions and the angle by which molecules are twisted changes linearly across the whole structure (see Fig. 1):

$$
\theta=\theta_{0}+\frac{2 \pi}{P} \cdot x
$$

where $\theta$-angle, $\theta_{0}$-anchoring condition, $P$-pitch

For the examined structure we assume electrical permittivity $\varepsilon$ in the following tensor form:

$$
\varepsilon=\left[\begin{array}{ccc}
\varepsilon_{\perp} & 0 & 0 \\
0 & \varepsilon_{\perp}+\Delta \varepsilon \cos ^{2} \theta & \Delta \varepsilon \sin \theta \cos \theta \\
0 & \Delta \varepsilon \sin \theta \cos \theta & \varepsilon_{\perp}+\Delta \varepsilon \sin ^{2} \theta
\end{array}\right]
$$

where $\Delta \varepsilon=\varepsilon_{\|}-\varepsilon_{\perp}, \theta$ - angle by which the molecules are twisted.

After simple algebraic operations six complex partial differential equations are obtained which are solved using a finite difference method. The great advantage of vectorial methods is that we obtain the values of all electric and magnetic components so we have information not only about light intensity but also about polarization. In our simulations Dirichlet boundary conditions are used. It means that the electric and magnetic fields vanish at the edges. However, such boundary conditions have a serious disadvantage as they act as a reflecting surface they are not so computing power consuming as TBC (Transparent Boundary Conditions) or PML (Perfectly Matched Layer)[7][8].

The material parameters used in simulations correspond to the 6CHBT nematic liquid crystal with a chiral doppant. Its ordinary and extraordinary refractive indices, at a temperature of $23^{\circ} \mathrm{C}$, have the following values[9][10]: $n_{o}=1,5144 n_{e}=1,6714$. In the following simulations we examine the structure of the cholesteric twisted through an angle of 360 degrees (see Fig. 1). The whole cell has $25 \mu \mathrm{m}$ or $50 \mu \mathrm{m}$ height and the light of a wavelength of $790 \mathrm{~nm}$ is propagated along $z$ axis. A Gaussian beam linearly polarized along y axis is launched into the structure: 


$$
\begin{aligned}
& \operatorname{Re}\left(E_{y}\right)=-E_{0} \exp \left(-\frac{x^{2}}{2 w_{0}{ }^{2}}-\frac{y^{2}}{2 w_{0}^{2}}\right) \\
& \operatorname{Re}\left(H_{x}\right)=\frac{\sqrt{\varepsilon_{22}}}{Z_{0}} E_{0} \exp \left(-\frac{x^{2}}{2 w_{0}{ }^{2}}-\frac{y^{2}}{2 w_{0}{ }^{2}}\right)
\end{aligned}
$$

where, $E_{0}-$ amplitude, $w_{0}=\frac{F W H M}{2 \sqrt{2 \ln 2}}-$ beam waist, $Z_{0}=\sqrt{\mu_{0} / \varepsilon_{0}} \approx 376,73 \Omega$ - impedance of vacuum [11].

Simulations were run for different position of launched beam and different FWHM. For all the following simulations (if not stated otherwise) $\theta_{0}=0$, and the angle $\theta$ is calculated form y axis.

In general, when talking about y-polarized light, the cholesteric in presented configuration can be described as a gradient waveguide with maximum refractive index at the center and gradually changing towards the edges. Starting from the center of the cell the refractive index decreases along the $\mathrm{x}$ axis and then increases and reaches its maximum at the edge.

The first presented results show a beam launched centrally into the structure of the cholesteric rotated through an angle of 360 degrees. The light propagates along the $\mathrm{z}$ axis.

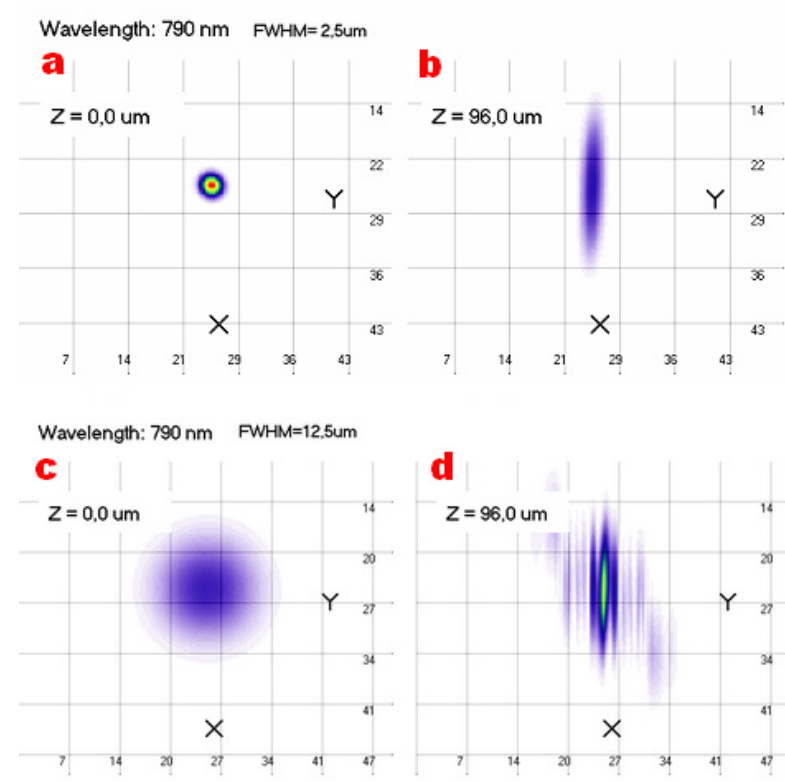

Fig.2 Light propagation in the structure $(50 \mu \mathrm{m})$ rotated through an angle of $360 \mathrm{deg}$. - intensity in the cross section at $z=0 \mu \mathrm{m}$, and $z=96 \mu \mathrm{m}$ for different beam width (a),(b) FWHM=2,5 $\mu \mathrm{m}$ (c),(d) $\mathrm{FWHM}=12,5 \mu \mathrm{m}$

For the beam of FWHM=2,5 $\mu$ diffraction can be only observed across the $\mathrm{y}$ axis and nearly no diffraction is observed across the $\mathrm{x}$ axis (see Fig. 2a,b ). It can be compared to the light propagation in a gradient waveguide. Light is guided in the area of a higher refractive index. For a higher width $(\mathrm{FWHM}=12,5 \mu \mathrm{m})$ the beam diffracts along the $y$ axis and focuses along the $\mathrm{x}$ axis (see Fig. 2c,d). It can be understood on the ground of the mode theory. For narrow beams only the fundamental mode is guided while for wider beams modes of higher order appear.

In the previous example the beam was launched at the center of the cholesteric. In the following example the beam is launched non-centrally. FWHM of the beam is $5 \mu \mathrm{m}$. At first, the light moves to the center of the structure. It is reasonable as polarization coincides with the direction of the molecules at the center. In other words, for y-polarized light there is a higher refractive index. When the light reaches the center of the cell, it splits into two separate beams. It is so because the moving beam changes slightly its polarization and moreover, as in the previous example, it diffracts along the $y$ axis.

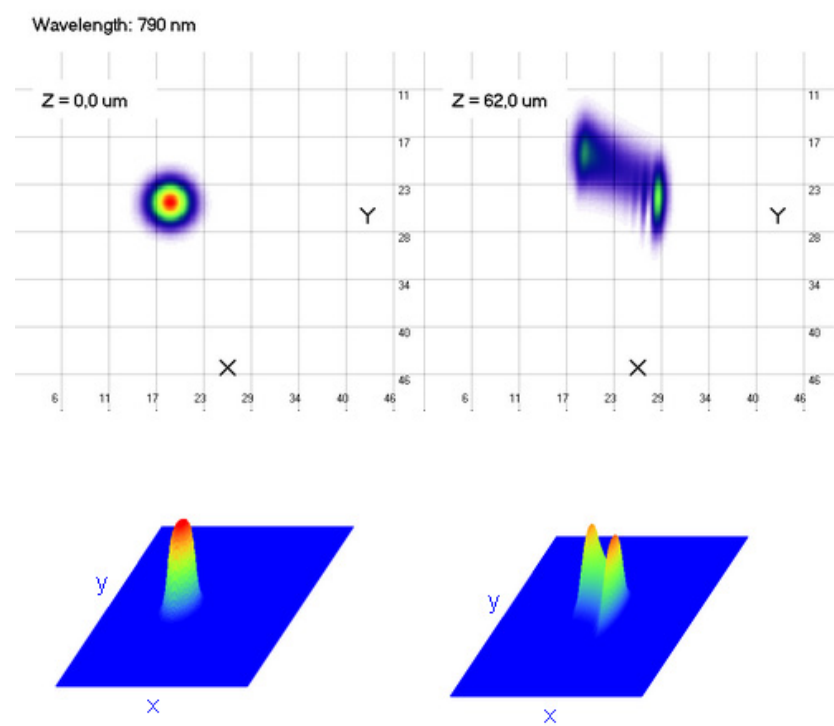

Fig. 3 Light propagation in the structure $(50 \mu \mathrm{m})$ rotated through an angle of 360 deg. Beam was launched non-centrally. Light intensity in cross section (xy plane) at $z=0 \mu \mathrm{m}$ (left) and $z=62 \mu \mathrm{m}$ (right). FWHM $=5 \mu \mathrm{m}$. Both graphs at the bottom are the tree-dimensional projections of the above results.

When the structure is rotated 360 degrees with a perpendicular anchoring condition $\left(\theta_{0}=90\right)$ the lowest refractive index is at the center and on the edges of the cell.

In such a situation the light launched at the center splits into two separate beams and then returns back towards the center (see. Fig. 4). It is clearly visible that the propagation of light is similar to the reflection from the surface of a lower refracting index. In fact, the cholesteric, as mentioned before, can be considered as a gradient waveguide. 


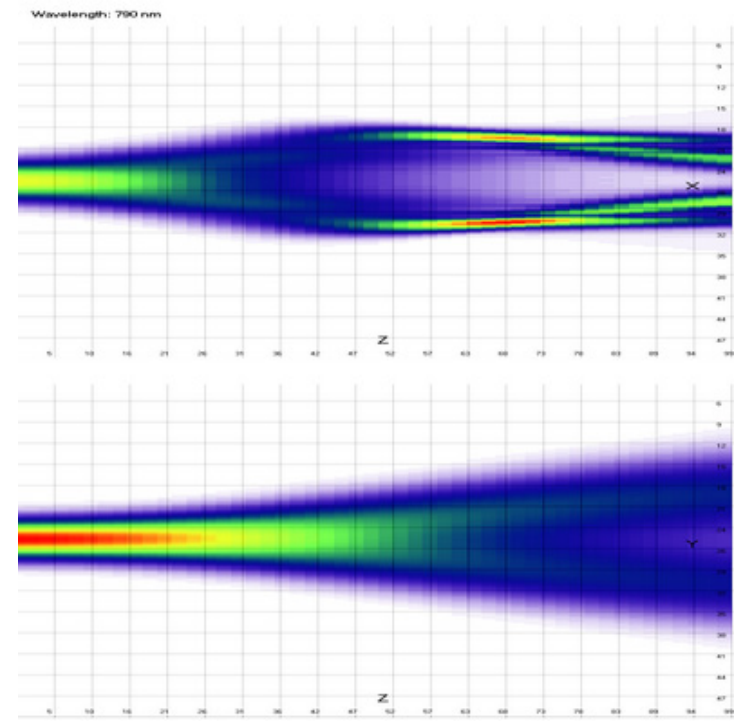

Fig. 4 Light propagation in the structure $(25 \mu \mathrm{m})$ rotated through an angle of $360 \mathrm{deg}$. with perpendicular anchoring condition $\left(\theta_{0}=90^{\circ}\right) \mathrm{Sum}$ intensity xz plane (top), sum intensity yz plane (bottom). FWHM=5 $\mu \mathrm{m}$.

The simulations were also made for the beam launched at some angle (up to $45 \mathrm{deg}$.) to the $\mathrm{x}$ and $\mathrm{y}$ axis. The beam bounces from one side to another. It can be also described as the propagation in a gradient waveguide with reflections at the edges (See Fig. 5).

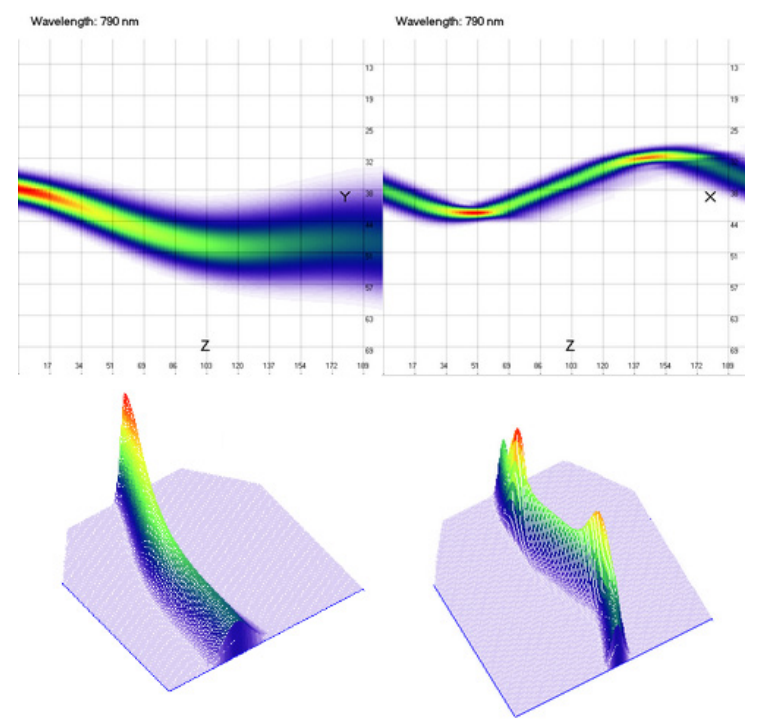

Fig. 5 Light propagation when the beam is launched at some acute angle. Light intensity yz plane (upper left), xz plane (upper right), 3D plots of light intensity of the above planes (at bottom).

All the simulations were carried out on the $\mathrm{PC}$ with Intel Pentium ${ }^{\circledR}$ IV $2,66 \mathrm{GHz}$ and 1,5GB RAM. The time of computation at a distance of $100 \mu \mathrm{m}$ was up to a few hours. The resolution was set to $0,25 \mu \mathrm{m}$ along the $\mathrm{x}$ and $\mathrm{y}$ axes and to $0,01 \mu \mathrm{m}$ along the $\mathrm{z}$ axis (see Fig. 6).

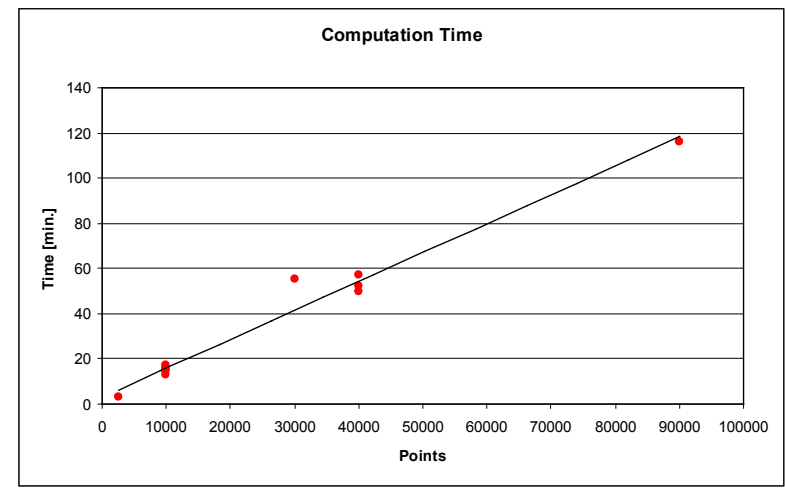

Fig. 6 Computation time (in minutes) in function of total points in the numerical grid.

Summarizing, the Full-Vector Beam Propagation Method can be used to simulate propagation in anisotropic media e.g. in cholesterics. Using Dirichlet boundary conditions is sufficient enough and the calculations take up to a few hours. Using this method it is possible to determine the parameters important in an experiment and applications. The presented results show that the position at which the beam is launched is crucial, as non-centrally launched beams tend to split into separate beams. Moreover, the beam must have the accurate width as too large beams are launched into different layers. Last but not least, the beam launched at some acute angle bounces from one side to another and can escape form the cell, so it is also very important for the beam to be propagated directly along the $\mathrm{z}$ axis.

\section{References}

[1] G. D. Ziogos, E. E. Kriezis, Modeling light propagation in liquid crystal devices with a 3-D full-vector finite-element beam propagation method, Opt. Quant. Electron 40, 10 (2008)

[2] I-C Khoo Liquid crystals John Wiley \& Sons, Inc (2007)

[3] U. A. Laudyn, M. Kwasny, M. A. Karpierz Nematicons in chiral nematic liquid crtystals, Appl. Phys. Lett. 94, 091110 (2009)

[4] U. A. Laudyn, M. Kwaśny, K. Jaworowicz, K. A. Rutkowska, M. A. Karpierz, G. Assanto Nematicons in twisted liquid crystals, Phot Lett. Poland, 1, 7-9 (2009)

[5] G. Assanto, M. Karpierz, Nematicons: self-localized beams in nematic liquid crystals, Liquid Crystals (2009)

[6] G. Assanto, M. Peccianti, C. Conti, Optical spatial solitons in nematic liquid crystals Opt. Photon. News 14, 44, (2003)

[7] J. P Berenger A perfectly matched layer for the absorption of electromagnetic waves, J. Comp. Phys. 114, (1994)

[8] D. S Katz, E.T. Thiele, A. Taflove Validation and extension to three dimensions of the Berenger PML absorbing boundary condition for FD-TD meshes, IEEE Microwave Guided Wave Lett. 4, (1994)

[9] J. Baran, Z. Raszewski, R. Dąbrowski, J. Kędzierski and J. Rutkowska, Mol. Cyst. Liq. Cyst. 123 (1985)

[10]R. Dabrowski, J. Dziaduszek and T. Szczuciński, Mol. Cyst. Liq. Cyst. 124, (1985)

[11]http://physics.nist.gov/cgi-bin/cuu/Value?eqz0 